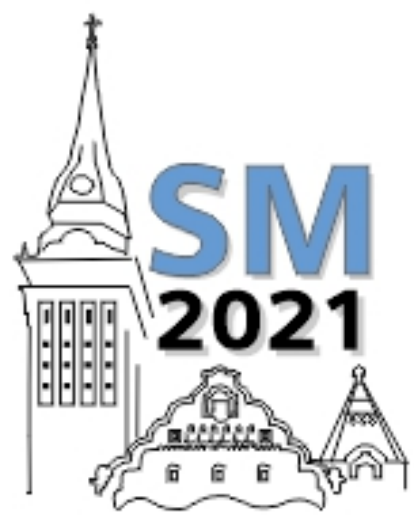

Dimitrije Gašić

The University of Novi Sad, Faculty of Economics in Subotica. Ph.D. student Novi Sad, The Republic of Serbia dimitrijegasic33@gmail.com 26th International Scientific Conference Strategic Management and Decision Support Systems in Strategic Management

21st May, 2021, Subotica, Republic of Serbia

\title{
THE INFLUENCE OF FLEXIBLE WORK ARRANGEMENTS ON THE CORPORATE EFFICIENCY DURING THE COVID-19 PANDEMIC IN THE REPUBLIC OF SERBIA
}

\begin{abstract}
Research Question: The author investigates the impact of the application of flexible work arrangements on the corporate efficiency of companies during the COVID - 19 pandemic in the Republic of Serbia. The main question is whether the application of flexible work arrangements has a positive impact on the efficiency of the corporation, its employees and how their application affects the business of companies in the current situation that has not passed any business sector around the world. Motivation: Based on the study by authors such as Bhattacharya et al. (2005), Austin-Egole et al. (2020), Berber \& Slavić (2019), the author plans to identify the impact of flexible work arrangements on corporate efficiency. The idea for the research arose due to the change in the way of doing business and the increasing application of flexible work arrangements in the modern business of almost every company around the world due to the outbreak of the COVID -19 pandemic. Data: The research was conducted at the end of 2020. and the beginning of 2021. on a sample of 219 employees who were greatly influenced by this pandemic in the business itself, which largely differs from the previous one. Data collection was done through an electronic questionnaire Google-Forms, based on which employees had the opportunity to answer questions with their electronic devices at any time, which is a great advantage especially in today's lifestyle where everything has become mobile and internet access enabled at any time wherever you are. Tools: To analyze the collected data, the SPSS (The Statistical Package for the Social Sciences) program was used. In his work, the author applied the following data processing techniques: T-test of independent samples, One-way ANOVA, and Spearman correlation. Findings: Based on the conducted research, the results indicate a positive relationship between flexible work arrangements and employee productivity. Contribution: The paper contributes to the literature by empirically examining the impact of certain factors on corporate efficiency and thus on the company's success in the Republic of Serbia during the COVID - 19 pandemic.
\end{abstract}

Keywords: COVID - 19 pandemic, flexible work arrangements, corporate efficiency, teleworking, Serbia.

\section{Introduction}

In their efforts to attract new and retain current talent in their companies, employers around the world are becoming more flexible so that employees can enjoy their work as much as possible and work hard, thus influencing the company's better success and remaining competitive in a harsh world. businesses where the goal is to survive, win, and improve your business, be better than others and conquer new markets around the world (Dalton \& Mesch, 1990; Barker, 1995; Valverde, Tregaskis, \& Brewster, 2000). Wright \& Snell (1998) defined flexibility as the ability of companies to reconfigure activities and resources quickly in response to environmental demands. The explanation for this followed given that the vast majority of companies are facing environments characterized by increasing dynamism and competition. In such an environment, the sustainability of companies can only be achieved if companies develop by applying flexibility in work. As a result, practitioners and academics have begun to consider flexibility as a strategic imperative (Ferris et al., 1998). In response to job change, companies have developed flexible work arrangements as work patterns that include modification of the regular workplace such as night work, weekend work, work from home, part-time work, teleworking, etc. (Stavrou, 2005; Coenen \& Kok, 2014), while giving employees the choice of when and where to work, how much 
work to do (Azar et al., 2018). Numerous scientific papers have confirmed that flexible work arrangements have a significant impact on organizational performance (Baltes et al., 1999; Beauregard \& Henry, 2009; Battisti \& Vallanti, 2013; Berkery et al., 2017; Stavrou, 2005). The paper is structured in three parts, the first part contains an overview of the relevant structure related to flexible work arrangements and the impact of flexible work arrangements on the corporate efficiency of companies. The second part refers to the research methodology where the questionnaire used during the research is described, how many parts the questionnaire consists of, questions for each part of the questionnaire, etc. In addition to the questionnaire, the sample also describes sample, in this part the sample of 219 respondents who completed the survey related to the application of flexible work arrangements for corporate efficiency due to the COVID-19 pandemic in the Republic of Serbia is described in detail. In the third part of the research work, the author presented in a table all the performed analyzes of data obtained through the SPSS program, and below the presented tables a detailed discussion of the obtained results was performed. After the presentation and discussion of the obtained results, concluding remarks follow in which the author makes observations and gives recommendations for future research. Due to the lack of literature and understanding of the impact of flexible work arrangements on corporate efficiency during the COVID19 pandemic in the Republic of Serbia, the paper seeks to clarify in more detail the impact of the pandemic on corporate efficiency. In this sense, this study aims to characterize the flexible work arrangements implemented in the Republic of Serbia in the context of the COVID - 19 pandemic.

\section{Theoretical background}

\subsection{Flexible work arrangements and teleworking}

Flexible work arrangements are practices such as "work from home", "work outside regular working hours", "reduced as well as extended contract hours", etc. (Den Dulk, Groeneveld, Ollier-Malaterre, \& Valcour, 2013, Stirpe \& ZárragaOberty, 2017). Companies around the world offer their employees the option of applying flexible work arrangements to balance their work and private lives but also to improve the business success of the company they work for (Richardson, \& McKenna, 2014). Flexible work arrangements can have multiple meanings around the world. According to Cranet International Research Methodology, some of the measures of FWAs are: Weekend work - Employees can extend working hours during the weekend or can work in 2 shifts, Saturday and Sunday with free time during the rest of the week. Work in shifts - Employees are replaced in job positions so that the Company can work longer than the working hours of individual employees. Part-time work - Employed workers regularly work less than a whole week. OvertimeEmployees work additional hours during the day, weekly, or over a year provided for in their employment contracts. Work from home - Employees work from home. Teleworking - Employees work separately from the office throughout the workweek, while maintaining an electronic presence in the office. Hourly contracts - Employees perform a predetermined number of working hours per year, with the division of hours being based either on an agreement between the employed workers and the employer or the basis of the last need for market demands. Division of labor - Division of labor two part-time employees share one full-time job. Flexible working hours - Employees work full time by being able to choose the start and end of working hours within the limits set by the company's management. Temporary employment - Employment is offered temporarily. Fixed-term contracts - Employees work under short-term or long-term employment contracts with a fixed duration (Stavrou, Parry \& Anderson, 2015.; Berber \& Slavić, 2019. p. 35).

\subsection{Relations between Flexible Work Arrangements and Job performance of employees.}

The relationship between flexible work arrangements and the business performance of employees has been examined in several different studies. Using social exchange theories (Blau, 1964), it has been found that improved work performance can be the result of employee-employer reciprocity (Golden, 2001, 2009; according to Kelliher \& Anderson, 2010) and where employees have had the option of using flexible work arrangements, they felt an obligation to the employer. A different but related perspective Konrad \& Mangel (2000) used Akerlof's (1982) theory of gift exchange that has a positive effect on the provision of work-life programs and worker productivity. The basis of the exchange of gifts is a situation in which the employer provides a "gift" to the employee by paying him wages or other benefits above what is required by the market and the employee will reciprocate with a "gift" of performance above the norm. In the case of informal flexible work arrangements (Atkinson \& Hall, 2009) observed that an informal work arrangement creates a sensible employee obligation and a consequent need for reciprocity that would lead to behavior considered to be valued by the manager who approved this type of arrangement as would work to provide extra effort. An important message for managers is that the role of flexible work arrangements can have a positive impact on organizational results (De Menezes \& Kelliher, 2016). Employers should find a way to implement all kinds of flexible work arrangements that generate positive organizational results such as turnover, less absenteeism, espionage among employees, and aimed at increased performance. This type of strategy should support the developing economy, especially in the Third World now that employee productivity and performance are declining. Unlike flexible work arrangements run by employees, arrangements that primarily benefit the employer especially traditional arrangements such as shift work, weekend work, fixed-term contracts, the annual number 
of hours is likely to create negative links with both financial and non-financial outcomes. Hence the importance for the creation and development of flexible work arrangements that meet the needs of employers and employees of the company, which would improve the quality of life of workers and organizational performance and this can never be overemphasized (Austin-Egole, EBJ \& Nwokorie, 2020. p. 56 .).

H1: Flexible work arrangements have a positive impact on the job performance of employees and the organizational success of the company.

In addition to the basic hypothesis, the paper will investigate the impact of flexible work arrangements according to public or private sector affiliation as well as the impact of the market that the company serves (locally, regionally, nationally, internationally, globally).

\section{Methodology 2.1. The questionnaire}

During the research process on the impact of flexible work arrangements on corporate efficiency during the COVID-19 pandemic in the Republic of Serbia, a three-part questionnaire was used. The first part refers to control questions: gender, age structure, level of education, position in the company, sector to which the company belongs by activities, size of organization, affiliation to public or private sector, market that the respondent company serves, as well as refers to the assessment of employee productivity levels. The second part deals with questions from Cranet's questionnaire regarding the application of flexible work arrangements. CRANET stands for The Cranfield Network on International Human Resource Management. The CRANET questionnaire was compiled by an international team of academics who have been researching human resource management since the late 1980s. The questionnaire consists of six parts and measures of numerous human resource management indicators. Cranet asked respondents to determine if there were formally twelve FVA practices in their workplace for any of the groups of employees. The second part regarding the application of flexible work arrangements consists of 10 questions that the respondents answered on a scale of $1-5(1=$ not used at all, $5=$ used extensively). Respondents were asked to indicate the extent to which flexible work arrangements are used in their company: weekend work, shift work, overtime, part-time work, flexible working hours, temporary work, teleworking / pre-COVID -19 pandemics), teleworking (during the COVID-19 pandemic) and teleworking after the COVID-19 pandemic). (Klindžić \& Marić, 2019). The third part of the question refers to the business performance of employees, this part consists of 5 questions: 1 . I always perform all the tasks listed in the job description, 2. I fulfill the formally required performance of this job, 3. I fulfill all responsibilities that the job requires of me, 4. I never neglect aspects of the job that I am required to perform, 5. I often fail to perform basic duties at work. (R). (Janssen \& Van Yperen, 2004). Respondents responded to the questions asked by ticking the boxes provided below each question. In response to the difficulty of measuring character and personality traits, Likert developed a procedure for measuring attitudes through a scale (Boone \& Boone, 2012, p. 1). Respondents answered the questions in the questionnaire based on the Likert scale in the range of 1 - $5(1=$ Strongly disagree, $5=$ Strongly agree $)$. The research was conducted through the electronic questionnaire "Google forms". The questionnaire used in this research is intended exclusively for employees. The link of the questionnaire was primarily distributed to people I know personally who move in the business world and who belong to various sectors than those in which the application of FWAs is most present such as in education, finance, and labor insurance, professional, scientific, innovative and technical activities, trade sector, etc. The electronic questionnaire was filled out by 219 employees throughout the Republic of Serbia, and data collection was performed from December 2020 to February 2021.

\subsection{The sample}

The research of the impact of flexible work arrangements on corporate efficiency during the COVID-19 pandemic in the Republic of Serbia was conducted using the electronic questionnaire "Google Forms". The questionnaire was completed by 219 employees throughout the Republic of Serbia. Data collection began in early December 2020 and ended in late February 2021. The main part of the sample consists of members of the female population with as many as $(57.99 \%)$, while the rest are respondents of the male population with (42.01\%). Examining the age structure of the respondents, we conclude that the largest part of the respondents belongs to the age between 25-34 years (36.99\%), while the smallest number of them belongs to the population older than 55 years of age $(7.76 \%)$. Regarding the level of education of respondents, the largest number of respondents completed master's studies $(36.07 \%)$, this percentage is close to the number of respondents who completed basic academic studies $(35.16 \%)$. One of the significant indicators is that out of a total of 219 respondents, as many as 25 of them completed doctoral studies $(11.42 \%)$, while the smallest number of respondents completed three-year vocational studies $(2.74 \%)$. Based on the completed questionnaires, the largest number of respondents belongs to professional workers $(53.42 \%)$, while the smallest number of them belong to manual workers (4.11\%). The largest number of respondents belongs to sector K. Financial and insurance activities (15.07\%), while the smallest number belongs to sector R. Arts, entertainment and recreation ( $0.46 \%)$. Based on the completed questionnaires, we conclude that the largest number of respondents work in a medium-sized organization (38.81\%), while the smallest number belongs to a large-sized organization $(26.03 \%)$. The main sample belongs to the private sector with as much as 
$(65.3 \%)$, while the rest of the respondents belong to the public sector $(34.7 \%)$. The majority of respondents answered that their company serves the national market (27.85\%), while the smallest number of them serve the global market (12.79\%). The majority of respondents answered that the headquarters of the company in which they work in the Republic of Serbia is even $(78.54 \%)$, while the smallest number of them answered that the headquarters of the company is in a non-EU country $(2.28 \%)$. The largest number of respondents stated that the company in which they work is a national company $(67.58 \%)$, while the smallest number of respondents stated that they work in a branch of a national company $(5.02 \%)$.

Table 1: Sample characteristics

\begin{tabular}{|c|c|c|c|c|c|}
\hline \multicolumn{6}{|c|}{ Grande } \\
\hline & & Frequency & Percent & Valid Percent & Cumulative Percent \\
\hline \multirow[t]{3}{*}{ Valid } & Male & 92 & 35.4 & 42.0 & 42.0 \\
\hline & Female & 127 & 48.8 & 58.0 & 100.0 \\
\hline & Total & 219 & 84.2 & 100.0 & \\
\hline Missing & System & 41 & 15.8 & & \\
\hline Total & & 260 & 100.0 & & \\
\hline \multicolumn{6}{|c|}{ Age } \\
\hline & & Frequency & Percent & Valid Percent & Cumulative Percent \\
\hline \multirow[t]{6}{*}{ Valid } & Less than 25 & 42 & 16.2 & 19.2 & 19.2 \\
\hline & $25-34$ & 81 & 31.2 & 37.0 & 56.2 \\
\hline & $35-44$ & 45 & 17.3 & 20.5 & 76.7 \\
\hline & $45-55$ & 34 & 13.1 & 15.5 & 92.2 \\
\hline & More than 55 & 17 & 6.5 & 7.8 & 100.0 \\
\hline & Total & 219 & 84.2 & 100.0 & \\
\hline Missing & System & 41 & 15.8 & & \\
\hline Total & & 260 & 100.0 & & \\
\hline \multicolumn{6}{|c|}{ Education } \\
\hline & & Frequency & Percent & Valid Percent & Cumulative Percent \\
\hline \multirow[t]{7}{*}{ Valid } & High School & 19 & 7.3 & 8.7 & 8.7 \\
\hline & Three-year vocational studies & 6 & 2.3 & 2.7 & 11.4 \\
\hline & Bachelor & 77 & 29.6 & 35.2 & 46.6 \\
\hline & Master study & 79 & 30.4 & 36.1 & 82.6 \\
\hline & Magistar & 13 & 5.0 & 5.9 & 88.6 \\
\hline & Ph.D. study & 25 & 9.6 & 11.4 & 100.0 \\
\hline & Total & 219 & 84.2 & 100.0 & \\
\hline Missing & System & 41 & 15.8 & & \\
\hline Total & & 260 & 100.0 & & \\
\hline \multicolumn{6}{|c|}{ Possition in Company } \\
\hline & & Frequency & Percent & Valid Percent & Cumulative Percent \\
\hline \multirow[t]{5}{*}{ Valid } & Manager & 60 & 23.1 & 27.4 & 27.4 \\
\hline & Professional worker & 117 & 45.0 & 53.4 & 80.8 \\
\hline & Administrative worker & 33 & 12.7 & 15.1 & 95.9 \\
\hline & Manuel worker & 9 & 3.5 & 4.1 & 100.0 \\
\hline & Total & 219 & 84.2 & 100.0 & \\
\hline Missing & System & 41 & 15.8 & & \\
\hline Total & & 260 & 100.0 & & \\
\hline \multicolumn{6}{|c|}{ Sector } \\
\hline & & Frequency & Percent & Valid Percent & Cumulative Percent \\
\hline \multirow[t]{18}{*}{ Valid } & Sector A. Agriculture, forestry and fishing & 4 & 1.5 & 1.8 & 1.8 \\
\hline & Sector C. Manufacturing & 24 & 9.2 & 11.0 & 12.8 \\
\hline & Sector D. Electricity, gas, steam and air conditioning supply & 4 & 1.5 & 1.8 & 14.6 \\
\hline & Sector F. Construction & 7 & 2.7 & 3.2 & 17.8 \\
\hline & Sector G. Wholesale trade, retail trade, repair of motor vehicles and motorcycles & 17 & 6.5 & 7.8 & 25.6 \\
\hline & Sector H. Transport and storage & 8 & 3.1 & 3.7 & 29.2 \\
\hline & Sector I. Accommodation and catering services & 5 & 1.9 & 2.3 & 31.5 \\
\hline & Sector J. Information and communication & 7 & 2.7 & 3.2 & 34.7 \\
\hline & Sector K. Financial and insurance activities & 33 & 12.7 & 15.1 & 49.8 \\
\hline & Sector M. Professional, scientific, innovation and technical activities & 27 & 10.4 & 12.3 & 62.1 \\
\hline & Sector N. Administrative and support service activities & 2 & 0.8 & 0.9 & 63.0 \\
\hline & Sector O. Public administration and defense, compulsory social security & 10 & 3.8 & 4.6 & 67.6 \\
\hline & Sector P. Education & 42 & 16.2 & 19.2 & 86.8 \\
\hline & Sector Q. Health and Social Welfare & 9 & 3.5 & 4.1 & 90.9 \\
\hline & Sector R. Arts, entertainment and recreation & 1 & 0.4 & 0.5 & 91.3 \\
\hline & Sector S. Tourism & 7 & 2.7 & 3.2 & 94.5 \\
\hline & Sector W. Other service activities & 12 & 4.6 & 5.5 & 100.0 \\
\hline & Total & 219 & 84.2 & 100.0 & \\
\hline Missing & System & 41 & 15.8 & & \\
\hline Total & & 260 & 100.0 & & \\
\hline \multicolumn{6}{|c|}{ The size of the organization } \\
\hline & & Frequency & Percent & Valid Percent & Cumulative Percent \\
\hline \multirow[t]{4}{*}{ Valid } & Small & 77 & 29.6 & 35.2 & 35.2 \\
\hline & Medium & 85 & 32.7 & 38.8 & 74.0 \\
\hline & Large & 57 & 21.9 & 26.0 & 100.0 \\
\hline & Total & 219 & 84.2 & 100.0 & \\
\hline Missing & System & 41 & 15.8 & & \\
\hline Total & & 260 & 100.0 & & \\
\hline
\end{tabular}




\begin{tabular}{|c|c|c|c|c|c|}
\hline \multicolumn{6}{|c|}{ Belonging to the public or private sector } \\
\hline & & Frequency & Percent & Valid Percent & Cumulative Percent \\
\hline \multirow[t]{3}{*}{ Valid } & Private sector & 143 & 55.0 & 65.3 & 65.3 \\
\hline & Public sector & 76 & 29.2 & 34.7 & 100.0 \\
\hline & Total & 219 & 84.2 & 100.0 & \\
\hline Missing & System & 41 & 15.8 & & \\
\hline Total & & 260 & 100.0 & & \\
\hline \multicolumn{6}{|c|}{ The market that your company serves } \\
\hline & & Frequency & Percent & Valid Percent & Cumulative Percent \\
\hline \multirow[t]{6}{*}{ Valid } & Local & 36 & 13.8 & 16.4 & 16.4 \\
\hline & Regional & 40 & 15.4 & 18.3 & 34.7 \\
\hline & National & 61 & 23.5 & 27.9 & 62.6 \\
\hline & International & 54 & 20.8 & 24.7 & 87.2 \\
\hline & Global & 28 & 10.8 & 12.8 & 100.0 \\
\hline & Total & 219 & 84.2 & 100.0 & \\
\hline Missing & System & 41 & 15.8 & & \\
\hline Total & & 260 & 100.0 & & \\
\hline \multicolumn{6}{|c|}{ Your company is headquartered in: } \\
\hline & & Frequency & Percent & Valid Percent & Cumulative Percent \\
\hline \multirow[t]{6}{*}{ Valid } & Republic of Serbia & 172 & 66.2 & 78.5 & 78.5 \\
\hline & EU & 29 & 11.2 & 13.2 & 91.8 \\
\hline & A non-EU country & 5 & 1.9 & 2.3 & 94.1 \\
\hline & USA & 7 & 2.7 & 3.2 & 97.3 \\
\hline & Other & 6 & 2.3 & 2.7 & 100.0 \\
\hline & Total & 219 & 84.2 & 100.0 & \\
\hline Missing & System & 41 & 15.8 & & \\
\hline Total & & 260 & 100.0 & & \\
\hline \multicolumn{6}{|c|}{ Your organization is: } \\
\hline & & Frequency & Percent & Valid Percent & Cumulative Percent \\
\hline \multirow[t]{5}{*}{ Valid } & National company & 148 & 56.9 & 67.6 & 67.6 \\
\hline & A subsidiary of a national company & 11 & 4.2 & 5.0 & 72.6 \\
\hline & An international company & 26 & 10.0 & 11.9 & 84.5 \\
\hline & A subsidiary of an international company & 34 & 13.1 & 15.5 & 100.0 \\
\hline & Total & 219 & 84.2 & 100.0 & \\
\hline Missing & System & 41 & 15.8 & & \\
\hline Total & & 260 & 100.0 & & \\
\hline
\end{tabular}

Source: Authors' calculations

\section{Results}

Based on the research conducted through the questionnaire on the impact of FWAs on corporate efficiency during the COVID-19 pandemic in the Republic of Serbia, primary data on respondents were obtained and the results are presented.

Table 2. T-test of independent samples of FWAs, level of productivity of employees, and job performance of employees according to their affiliation with the public or private sector.

\begin{tabular}{|c|c|c|c|c|c|}
\hline & Belonging to the public or private sector & $\mathbf{N}$ & Mean & Std. Deviation & Std. Error Mean \\
\hline \multirow{2}{*}{ Weekend work } & \begin{tabular}{|l|} 
Private sector \\
\end{tabular} & 143 & 3.11 & 1.511 & 0.126 \\
\hline & Public sector & 76 & 3.41 & 1.368 & 0.157 \\
\hline \multirow{2}{*}{ Work in shifts } & Private sector & 143 & 3.1 & 1.677 & 0.14 \\
\hline & Public sector & 76 & 3.58 & 1.407 & 0.161 \\
\hline \multirow{2}{*}{ Overtime } & Private sector & 143 & 3.68 & 1.242 & 0.104 \\
\hline & Public sector & 76 & 3.87 & 1.17 & 0.134 \\
\hline \multirow{2}{*}{ Part time job } & Private sector & 143 & 3.06 & 1.507 & 0.126 \\
\hline & Public sector & 76 & 3.53 & 1.409 & 0.162 \\
\hline \multirow{2}{*}{ Flexible working hours } & Private sector & 143 & 3.79 & 1.326 & 0.111 \\
\hline & Public sector & 76 & 3.7 & 1.461 & 0.168 \\
\hline \multirow{2}{*}{ Temporary employment } & Private sector & 143 & 3.36 & 1.437 & 0.12 \\
\hline & Public sector & 76 & 3.64 & 1.293 & 0.148 \\
\hline \multirow{2}{*}{ Teleworking before COVID - 19 pandemic } & Private sector & 143 & 2.69 & 1.456 & 0.122 \\
\hline & Public sector & 76 & 2.32 & 1.213 & 0.139 \\
\hline \multirow{2}{*}{ Teleworking during the COVID - 19 pandemic } & Private sector & 143 & 3.9 & 1.334 & 0.112 \\
\hline & Public sector & 76 & 4.21 & 1.111 & 0.127 \\
\hline \multirow{2}{*}{ Teleworking after COVID - 19 pandemic } & Private sector & 143 & 3.66 & 1.379 & 0.115 \\
\hline & Public sector & 76 & 4 & 1.2 & 0.138 \\
\hline \multirow{2}{*}{ The level of productivity of employees } & Private sector & 143 & 3.84 & 1.197 & 0.1 \\
\hline & Public sector & 76 & 3.76 & 1.274 & 0.146 \\
\hline \multirow{2}{*}{ Job performance of employees } & Private sector & 143 & 4.606 & 0.462 & 0.0386 \\
\hline & Public sector & 75 & 4.573 & 0.4654 & 0.0537 \\
\hline
\end{tabular}

Source: Authors' calculation 
The table shows the results of statistical groups according to their affiliation with the public or private sector as well as according to the categories important for corporate efficiency. Based on the conducted research, we conclude that the majority of respondents belong to the private sector 143 employees $(65.3 \%)$, while the rest of the respondents work in a company belonging to the public sector 76 employees $(34.7 \%)$. The T-test is a statistical procedure used to test the significance of the difference between two samples. We compare their arithmetic means. The T-test is one of the most commonly used tests of statistical hypotheses in studies of numerous scientific studies (Kim, 2015).

Table 2.1. Review of T-test of independent samples of FWAs, level of productivity of employees, and job performance of employees according to their affiliation with the public or private sector.

\begin{tabular}{|c|c|c|c|c|c|c|c|c|c|c|}
\hline & & \multicolumn{2}{|c|}{ Levene's Test for Equality of Variances } & \multirow[b]{2}{*}{$\mathbf{t}$} & \multirow[b]{2}{*}{ df } & \multirow[b]{2}{*}{$\begin{array}{l}\text { Sig. (2- } \\
\text { tailed) }\end{array}$} & \multicolumn{2}{|c|}{$\mathrm{t}$-test for Equality of Means } & \multicolumn{2}{|c|}{$95 \%$ Confidence Interval of the Difference } \\
\hline & & $\mathbf{F}$ & Sig. & & & & $\begin{array}{c}\text { Mean } \\
\text { Difference }\end{array}$ & $\begin{array}{l}\text { Std. Error } \\
\text { Difference }\end{array}$ & Lower & Upper \\
\hline \multirow{2}{*}{ Weekend work } & Equal variances assumed & 2.349 & 0.127 & -1.425 & 217 & 0.156 & -0.296 & 0.208 & -0.705 & 0.113 \\
\hline & Equal variances not assumed & & & -1.469 & 166.762 & 0.144 & -0.296 & 0.201 & -0.694 & 0.102 \\
\hline \multirow{2}{*}{ Work in shifts } & Equal variances assumed & 12.611 & 0 & -2.102 & 217 & 0.037 & -0.474 & 0.226 & -0.919 & -0.029 \\
\hline & Equal variances not assumed & & & -2.217 & 177.537 & 0.028 & -0.474 & 0.214 & -0.896 & -0.052 \\
\hline \multirow{2}{*}{ Overtime } & Equal variances assumed & 0.443 & 0.506 & -1.1 & 217 & 0.273 & -0.19 & 0.173 & -0.531 & 0.151 \\
\hline & Equal variances not assumed & & & -1.12 & 161.236 & 0.264 & -0.19 & 0.17 & -0.525 & 0.145 \\
\hline \multirow{2}{*}{ Part time job } & Equal variances assumed & 0.654 & 0.42 & -2.215 & 217 & 0.028 & -0.463 & 0.209 & -0.876 & -0.051 \\
\hline & Equal variances not assumed & & & -2.261 & 162.207 & 0.025 & -0.463 & 0.205 & -0.868 & -0.059 \\
\hline \multirow{2}{*}{ Flexible working hours } & Equal variances assumed & 2.321 & 0.129 & 0.476 & 217 & 0.635 & 0.093 & 0.195 & -0.292 & 0.477 \\
\hline & Equal variances not assumed & & & 0.462 & 140.817 & 0.645 & 0.093 & 0.201 & -0.304 & 0.49 \\
\hline \multirow{2}{*}{ Temporary employment } & Equal variances assumed & 3.081 & 0.081 & -1.426 & 217 & 0.155 & -0.281 & 0.197 & -0.67 & 0.107 \\
\hline & Equal variances not assumed & & & -1.473 & 167.589 & 0.143 & -0.281 & 0.191 & -0.658 & 0.096 \\
\hline \multirow{2}{*}{ Teleworking before COVID - 19 pandemic } & Equal variances assumed & 11.33 & 0.001 & 1.891 & 217 & 0.06 & 0.37 & 0.195 & -0.016 & 0.755 \\
\hline & Equal variances not assumed & & & 1.999 & 178.455 & 0.047 & 0.37 & 0.185 & 0.005 & 0.734 \\
\hline \multirow{2}{*}{ Teleworking during the COVID - 19 pandemic } & Equal variances assumed & 5.757 & 0.017 & -1.723 & 217 & 0.086 & -0.308 & 0.179 & -0.661 & 0.044 \\
\hline & Equal variances not assumed & & & -1.821 & 178.526 & 0.07 & -0.308 & 0.169 & -0.643 & 0.026 \\
\hline \multirow{2}{*}{ Teleworking after COVID - 19 pandemic } & Equal variances assumed & 4.693 & 0.031 & -1.792 & 217 & 0.075 & -0.336 & 0.187 & -0.705 & 0.034 \\
\hline & Equal variances not assumed & & & -1.869 & 172.337 & 0.063 & -0.336 & 0.18 & -0.69 & 0.019 \\
\hline \multirow{2}{*}{ The level of productivity of employees } & Equal variances assumed & 0.336 & 0.563 & 0.437 & 217 & 0.662 & 0.076 & 0.174 & -0.266 & 0.418 \\
\hline & Equal variances not assumed & & & 0.429 & 144.972 & 0.668 & 0.076 & 0.177 & -0.274 & 0.426 \\
\hline \multirow{2}{*}{ Job performance of employees } & Equal variances assumed & 0.745 & 0.389 & 0.489 & 216 & 0.626 & 0.0323 & 0.066 & -0.0979 & 0.1624 \\
\hline & Equal variances not assumed & & & 0.487 & 149.475 & 0.627 & 0.0323 & 0.0662 & -0.0985 & 0.163 \\
\hline
\end{tabular}

Source: Authors' calculation

Based on the conducted T-test of independent samples, the results of public or private sector testing were compared and based on the analysis of data in the SPSS program, the results showed that in "Work in shifts", "Part-time work" as well as in "Teleworking before the COVID-19 pandemic" there are statistically significant differences because of the value of Sig. (2-tailed) less than 0.05. According to the results of the T-test, it is evident that there are differences between companies in the public and private sector in terms of the use of shift work, where companies from the public sector use this type of FWAs $(\mathrm{M}=3.58)$, compared to the private sector. $(\mathrm{M}=3.1)$. Also in the use of part-time work, where companies from the public sector use this type of flexible work arrangement to a greater extent $(\mathrm{M}=3.53)$, compared to the private sector $(\mathrm{M}=3.06)$. In teleworking before the COVID-19 pandemic, there are differences between public and private sector companies where private sector companies make greater use of this type of arrangement $(\mathrm{M}=2.69)$ compared to the public sector $(\mathrm{M}=2.32)$. 
Table 3. Overview of the dimension of FWAs, level of productivity of employees, and job performance of employees according to the market that the company serves.

\begin{tabular}{|c|c|c|c|c|c|c|c|c|c|}
\hline & & \multirow{2}{*}{$\mathbf{N}$} & \multirow{2}{*}{ Mean } & \multirow{2}{*}{ Std. Deviation } & \multirow{2}{*}{$\begin{array}{l}\text { Std. } \\
\text { Error }\end{array}$} & \multicolumn{2}{|c|}{$\begin{array}{l}95 \% \text { Confidence Interval } \\
\text { for Mean } \\
\end{array}$} & \multirow{2}{*}{ Min } & \multirow{2}{*}{ Max } \\
\hline & & & & & & $\begin{array}{l}\text { Lower } \\
\text { Bound }\end{array}$ & Upper Bound & & \\
\hline \multirow{6}{*}{ Weekend work } & Local & 36 & 2.97 & 1.558 & 0.26 & 2.45 & 3.5 & 1 & 5 \\
\hline & \begin{tabular}{|l|} 
Regional \\
\end{tabular} & 40 & 3.18 & 1.483 & 0.234 & 2.7 & 3.65 & 1 & 5 \\
\hline & National & 61 & 3.8 & 1.138 & 0.146 & 3.51 & 4.09 & 1 & 5 \\
\hline & International & 54 & 2.98 & 1.498 & 0.204 & 2.57 & 3.39 & 1 & 5 \\
\hline & \begin{tabular}{|l|} 
Global \\
\end{tabular} & 28 & 2.79 & 1.595 & 0.301 & 2.17 & 3.4 & 1 & 5 \\
\hline & Total & 219 & 3.22 & 1.464 & 0.099 & 3.02 & 3.41 & 1 & 5 \\
\hline \multirow{6}{*}{ Work in shifts } & Local & 36 & 3.28 & 1.632 & 0.272 & 2.73 & 3.83 & 1 & 5 \\
\hline & \begin{tabular}{|l|} 
Regional \\
\end{tabular} & 40 & 3.3 & 1.713 & 0.271 & 2.75 & 3.85 & 1 & 5 \\
\hline & \begin{tabular}{|l|} 
National \\
\end{tabular} & 61 & 3.49 & 1.422 & 0.182 & 3.13 & 3.86 & 1 & 5 \\
\hline & \begin{tabular}{|l|} 
International \\
\end{tabular} & 54 & 3.22 & 1.598 & 0.217 & 2.79 & 3.66 & 1 & 5 \\
\hline & Global & 28 & 2.86 & 1.799 & 0.34 & 2.16 & 3.55 & 1 & 5 \\
\hline & Total & 219 & 3.27 & 1.602 & 0.108 & 3.06 & 3.49 & 1 & 5 \\
\hline \multirow{6}{*}{ Overtime } & Local & 36 & 3.5 & 1.207 & 0.201 & 3.09 & 3.91 & 1 & 5 \\
\hline & Regional & 40 & 3.6 & 1.194 & 0.189 & 3.22 & 3.98 & 1 & 5 \\
\hline & \begin{tabular}{|l|} 
National \\
\end{tabular} & 61 & 4.07 & 1.109 & 0.142 & 3.78 & 4.35 & 1 & 5 \\
\hline & International & 54 & 3.7 & 1.207 & 0.164 & 3.37 & 4.03 & 1 & 5 \\
\hline & Global & 28 & 3.64 & 1.446 & 0.273 & 3.08 & 4.2 & 1 & 5 \\
\hline & Total & 219 & 3.74 & 1.219 & 0.082 & 3.58 & 3.91 & 1 & 5 \\
\hline & Local & 36 & 3.17 & 1.521 & 0.254 & 2.65 & 3.68 & 1 & 5 \\
\hline & Regional & 40 & 2.8 & 1.454 & 0.23 & 2.34 & 3.26 & 1 & 5 \\
\hline Part time iob & National & 61 & 3.54 & 1.49 & 0.191 & 3.16 & 3.92 & 1 & 5 \\
\hline Part time job & International & 54 & 3.24 & 1.453 & 0.198 & 2.84 & 3.64 & 1 & 5 \\
\hline & Global & 28 & 3.21 & 1.524 & 0.288 & 2.62 & 3.81 & 1 & 5 \\
\hline & Total & 219 & 3.23 & 1.491 & 0.101 & 3.03 & 3.43 & 1 & 5 \\
\hline & Local & 36 & 3.33 & 1.434 & 0.239 & 2.85 & 3.82 & 1 & 5 \\
\hline & \begin{tabular}{|l|} 
Regional \\
\end{tabular} & 40 & 3.05 & 1.535 & 0.243 & 2.56 & 3.54 & 1 & 5 \\
\hline Flexible working hours & \begin{tabular}{|l|} 
National \\
\end{tabular} & 61 & 4.33 & 1.091 & 0.14 & 4.05 & 4.61 & 1 & 5 \\
\hline Flexible working hours & International & 54 & 3.85 & 1.25 & 0.17 & 3.51 & 4.19 & 1 & 5 \\
\hline & Global & 28 & 3.86 & 1.268 & 0.24 & 3.37 & 4.35 & 1 & 5 \\
\hline & Total & 219 & 3.75 & 1.369 & 0.093 & 3.57 & 3.94 & 1 & 5 \\
\hline & Local & 36 & 2.94 & 1.308 & 0.218 & 2.5 & 3.39 & 1 & 5 \\
\hline & \begin{tabular}{|l|} 
Regional \\
\end{tabular} & 40 & 2.93 & 1.623 & 0.257 & 2.41 & 3.44 & 1 & 5 \\
\hline Temporary emplayment & \begin{tabular}{|l|} 
National \\
\end{tabular} & 61 & 3.95 & 1.146 & 0.147 & 3.66 & 4.24 & 1 & 5 \\
\hline Temporary empioyment & International & 54 & 3.54 & 1.342 & 0.183 & 3.17 & 3.9 & 1 & 5 \\
\hline & Global & 28 & 3.61 & 1.37 & 0.259 & 3.08 & 4.14 & 1 & 5 \\
\hline & Total & 219 & 3.45 & 1.395 & 0.094 & 3.27 & 3.64 & 1 & 5 \\
\hline & Local & 36 & 3 & 1.474 & 0.246 & 2.5 & 3.5 & 1 & 5 \\
\hline & Regional & 40 & 2.25 & 1.548 & 0.245 & 1.75 & 2.75 & 1 & 5 \\
\hline Teleworkino before COVD-10 nandemic & \begin{tabular}{|l|} 
National \\
\end{tabular} & 61 & 2.31 & 1.148 & 0.147 & 2.02 & 2.61 & 1 & 5 \\
\hline Teleworking betore COVID - 19 pandemic & International & 54 & 2.46 & 1.284 & 0.175 & 2.11 & 2.81 & 1 & 5 \\
\hline & Global & 28 & 3.25 & 1.481 & 0.28 & 2.68 & 3.82 & 1 & 5 \\
\hline & Total & 219 & 2.57 & 1.394 & 0.094 & 2.39 & 2.76 & 1 & 5 \\
\hline & Local & 36 & 3.86 & 1.291 & 0.215 & 3.42 & 4.3 & 1 & 5 \\
\hline & Regional & 40 & 3.38 & 1.58 & 0.25 & 2.87 & 3.88 & 1 & 5 \\
\hline Teleworkino during the COYID - 19 andemic & \begin{tabular}{|l|} 
National \\
\end{tabular} & 61 & 4.34 & 0.929 & 0.119 & 4.11 & 4.58 & 1 & 5 \\
\hline Teleworking during the COVID - 19 pandemic & International & 54 & 3.93 & 1.257 & 0.171 & 3.58 & 4.27 & 1 & 5 \\
\hline & \begin{tabular}{|l} 
Global \\
\end{tabular} & 28 & 4.54 & 0.999 & 0.189 & 4.15 & 4.92 & 1 & 5 \\
\hline & Total & 219 & 4.01 & 1.267 & 0.086 & 3.84 & 4.18 & 1 & 5 \\
\hline & Local & 36 & 3.5 & 1.342 & 0.224 & 3.05 & 3.95 & 1 & 5 \\
\hline & Regional & 40 & 2.93 & 1.526 & 0.241 & 2.44 & 3.41 & 1 & 5 \\
\hline Teleworkino after COVD - 19 nandemic & National & 61 & 4.23 & 1.071 & 0.137 & 3.96 & 4.5 & 1 & 5 \\
\hline Teleworking after COVID - 19 pandemic & International & 54 & 3.78 & 1.254 & 0.171 & 3.44 & 4.12 & 1 & 5 \\
\hline & Global & 28 & 4.25 & 1.11 & 0.21 & 3.82 & 4.68 & 1 & 5 \\
\hline & Total & 219 & 3.76 & 1.337 & 0.09 & 3.58 & 3.94 & 1 & 5 \\
\hline & Local & 36 & 3.64 & 1.268 & 0.211 & 3.21 & 4.07 & 1 & 5 \\
\hline & Regional & 40 & 3.35 & 1.252 & 0.198 & 2.95 & 3.75 & 1 & 5 \\
\hline The lovel of nroductivity of omplovees & National & 61 & 4.16 & 1.113 & 0.143 & 3.88 & 4.45 & 1 & 5 \\
\hline The level of productivity of employees & International & 54 & 3.7 & 1.176 & 0.16 & 3.38 & 4.02 & 1 & 5 \\
\hline & Global & 28 & 4.14 & 1.208 & 0.228 & 3.67 & 4.61 & 1 & 5 \\
\hline & Total & 219 & 3.81 & 1.222 & 0.083 & 3.65 & 3.98 & 1 & 5 \\
\hline & Local & 36 & 4.539 & 0.5277 & 0.0879 & 4.36 & 4.717 & 3 & 5 \\
\hline & \begin{tabular}{|l|} 
Regional \\
\end{tabular} & 40 & 4.505 & 0.4391 & 0.0694 & 4.365 & 4.645 & 3.6 & 5 \\
\hline Joh nerformance of employees & \begin{tabular}{|l|} 
National \\
\end{tabular} & 61 & 4.718 & 0.3677 & 0.0471 & 4.624 & 4.812 & 3.6 & 5 \\
\hline Job performance of employees & International & 54 & 4.574 & 0.49 & 0.0667 & 4.44 & 4.708 & 2.6 & 5 \\
\hline & Global & 28 & 4.564 & 0.5049 & 0.0954 & 4.368 & 4.76 & 3.2 & 5 \\
\hline & Total & 219 & 4.595 & 0.4613 & 0.0312 & 4.533 & 4.656 & 2.6 & 5 \\
\hline
\end{tabular}

Source: Authors' calculation

The table shows the results of the statistical group according to the market they serve, as well as 11 categories important for corporate efficiency. Out of a total of 219 respondents, the largest number of them belong to the company that serves the "National Market" 61 (27.9\%), followed by "International" 54 (24.7\%), "Regional" 40 (18.3\%), "Local" 36 (16.4\%) and the smallest number of them belongs to the company that serves the "Global Market" 28 (12.8\%). 
Table 3.1. Test of Homogeneity of Variances FWAs, level of productivity of employees, and job performance of employees according to the market served by the company.

\begin{tabular}{|c|c|c|c|c|c|}
\hline & & Levene Statistic & df1 & df2 & Sig. \\
\hline \multirow{4}{*}{ Weekend work } & Based on Mean & \begin{tabular}{|l|}
3.882 \\
\end{tabular} & 4 & 214 & 0.005 \\
\hline & Based on Median & 4.598 & 4 & 214 & 0.001 \\
\hline & Based on Median and with adjusted df & 4.598 & 4 & 209.685 & 0.001 \\
\hline & Based on trimmed mean & 4.305 & 4 & 214 & 0.002 \\
\hline \multirow{4}{*}{ Work in shifts } & Based on Mean & 2.427 & 4 & 214 & 0.049 \\
\hline & Based on Median & 1.806 & 4 & 214 & 0.129 \\
\hline & Based on Median and with adjusted df & 1.806 & 4 & 199.516 & 0.129 \\
\hline & Based on trimmed mean & 2.513 & 4 & 214 & 0.043 \\
\hline \multirow{4}{*}{ Overtime } & Based on Mean & 2.405 & 4 & 214 & 0.051 \\
\hline & Based on Median & 1.321 & 4 & 214 & 0.263 \\
\hline & Based on Median and with adjusted df & 1.321 & 4 & 210.617 & 0.263 \\
\hline & Based on trimmed mean & 2.07 & 4 & 214 & 0.086 \\
\hline \multirow{4}{*}{ Part time job } & Based on Mean & 0.197 & 4 & 214 & 0.94 \\
\hline & Based on Median & 0.164 & 4 & 214 & 0.956 \\
\hline & Based on Median and with adjusted df & 0.164 & 4 & 207.606 & 0.956 \\
\hline & Based on trimmed mean & 0.177 & 4 & 214 & 0.95 \\
\hline \multirow{4}{*}{ Flexible working hours } & Based on Mean & 3.752 & 4 & 214 & 0.006 \\
\hline & Based on Median & 3.935 & 4 & 214 & 0.004 \\
\hline & Based on Median and with adjusted df & 3.935 & 4 & 193.656 & 0.004 \\
\hline & Based on trimmed mean & 4.055 & 4 & 214 & 0.003 \\
\hline \multirow{4}{*}{ Temporary employment } & Based on Mean & 4.53 & 4 & 214 & 0.002 \\
\hline & Based on Median & 3.743 & 4 & 214 & 0.006 \\
\hline & Based on Median and with adjusted df & 3.743 & 4 & 198.095 & 0.006 \\
\hline & Based on trimmed mean & 4.596 & 4 & 214 & 0.001 \\
\hline \multirow{4}{*}{ Teleworking before COVID - 19 pandemic } & Based on Mean & 4.113 & 4 & 214 & 0.003 \\
\hline & Based on Median & 3.794 & 4 & 214 & 0.005 \\
\hline & Based on Median and with adjusted df & 3.794 & 4 & 190.838 & 0.005 \\
\hline & Based on trimmed mean & 4.241 & 4 & 214 & 0.003 \\
\hline \multirow{4}{*}{ Teleworking during the COVID - 19 pandemic } & Based on Mean & 7.199 & 4 & 214 & 0 \\
\hline & Based on Median & 4.821 & 4 & 214 & 0.001 \\
\hline & Based on Median and with adjusted df & 4.821 & 4 & 196.236 & 0.001 \\
\hline & Based on trimmed mean & 6.97 & 4 & 214 & 0 \\
\hline \multirow{4}{*}{ Teleworking after COVID - 19 pandemic } & Based on Mean & 2.909 & 4 & 214 & 0.023 \\
\hline & Based on Median & 2.358 & 4 & 214 & 0.055 \\
\hline & Based on Median and with adjusted df & 2.358 & 4 & 197.513 & 0.055 \\
\hline & Based on trimmed mean & 2.965 & 4 & 214 & 0.021 \\
\hline \multirow{4}{*}{ The level of productivity of employees } & Based on Mean & 0.907 & 4 & 214 & 0.46 \\
\hline & Based on Median & 0.231 & 4 & 214 & 0.921 \\
\hline & Based on Median and with adjusted df & 0.231 & 4 & 191.166 & 0.921 \\
\hline & Based on trimmed mean & 0.809 & 4 & 214 & 0.521 \\
\hline \multirow{4}{*}{ Job performance of employees } & Based on Mean & 2.564 & 4 & 214 & 0.039 \\
\hline & Based on Median & 1.8 & 4 & 214 & 0.13 \\
\hline & Based on Median and with adjusted df & 1.8 & 4 & 181.339 & 0.131 \\
\hline & Based on trimmed mean & 2.611 & 4 & 214 & 0.037 \\
\hline
\end{tabular}

Source: Authors' calculation

The test of Homogeneity of Variances shows whether the assumption of variance homogeneity is violated. If the value of Sig. less than 0.05, homogeneity is impaired (Conover, Johnson \& Johnson, 1981). Based on the conducted test of homogeneity of variance, we conclude that in "Weekend work", "Work in shifts", "Flexible working hours", Temporary work "," Teleworking before COVID-19 pandemic", "Teleworking during the COVID-19 pandemic", "Teleworking after the COVID-19 pandemic" as well as in "Job performance of employees" the homogeneity of variance is disturbed. 
Table 3.2. Presentation of the dimension of FWAs, level of productivity of employees and job performance of employees, ANOVA test according to the market that the company serves.

\begin{tabular}{|c|c|c|c|c|c|c|}
\hline & & Sum of Squares & df & Mean Square & $\mathbf{F}$ & Sig. \\
\hline \multirow{3}{*}{ Weekend work } & Between Groups & 31.397 & 4 & 7.849 & 3.852 & 0.005 \\
\hline & Within Groups & 436.082 & 214 & 2.038 & & \\
\hline & Total & 467.479 & 218 & & & \\
\hline \multirow{3}{*}{ Work in shifts } & Between Groups & 7.932 & 4 & 1.983 & 0.769 & 0.546 \\
\hline & Within Groups & 551.63 & 214 & 2.578 & & \\
\hline & Total & 559.562 & 218 & & & \\
\hline \multirow{3}{*}{ Overtime } & Between Groups & 9.655 & 4 & 2.414 & 1.645 & 0.164 \\
\hline & Within Groups & 314.026 & 214 & 1.467 & & \\
\hline & Total & 323.68 & 218 & & & \\
\hline \multirow{3}{*}{ Part time job } & Between Groups & 13.452 & 4 & 3.363 & 1.528 & 0.195 \\
\hline & \begin{tabular}{|l} 
Within Groups \\
\end{tabular} & 471.132 & 214 & 2.202 & & \\
\hline & Total & 484.584 & 218 & & & \\
\hline \multirow{3}{*}{ Flexible working hours } & Between Groups & 47.099 & 4 & 11.775 & 6.969 & 0 \\
\hline & \begin{tabular}{|l} 
Within Groups \\
\end{tabular} & 361.586 & 214 & 1.69 & & \\
\hline & Total & 408.685 & 218 & & & \\
\hline \multirow{3}{*}{ Temporary employment } & Between Groups & 36.626 & 4 & 9.156 & 5.055 & 0.001 \\
\hline & Within Groups & 387.621 & 214 & 1.811 & & \\
\hline & Total & 424.247 & 218 & & & \\
\hline \multirow{3}{*}{ Teleworking before COVID - 19 pandemic } & Between Groups & 28.395 & 4 & 7.099 & 3.843 & 0.005 \\
\hline & \begin{tabular}{|l|} 
Within Groups \\
\end{tabular} & 395.258 & 214 & 1.847 & & \\
\hline & Total & 423.653 & 218 & & & \\
\hline \multirow{3}{*}{ Teleworking during the COVID - 19 pandemic } & Between Groups & 31.863 & 4 & 7.966 & 5.359 & 0 \\
\hline & Within Groups & 318.119 & 214 & 1.487 & & \\
\hline & Total & 349.982 & 218 & & & \\
\hline \multirow{3}{*}{ Teleworking after COVID - 19 pandemic } & Between Groups & 50.508 & 4 & 12.627 & 7.968 & 0 \\
\hline & Within Groups & 339.145 & 214 & 1.585 & & \\
\hline & Total & 389.653 & 218 & & & \\
\hline \multirow{3}{*}{ The level of productivity of employees } & Between Groups & 20.87 & 4 & 5.218 & 3.667 & 0.007 \\
\hline & \begin{tabular}{|l|} 
Within Groups \\
\end{tabular} & 304.454 & 214 & 1.423 & & \\
\hline & Total & 325.324 & 218 & & & \\
\hline \multirow{3}{*}{ Job performance of employees } & Between Groups & 1.411 & 4 & 0.353 & 1.678 & 0.156 \\
\hline & Within Groups & 44.983 & 214 & 0.21 & & \\
\hline & Total & 46.393 & 218 & & & \\
\hline
\end{tabular}

Source: Authors' calculation

Based on the conducted One-way ANOVA test shown in the table above, we conclude that there are statistically significant differences between "The level of productivity of employees" according to the market that the company serves.

Table 3.3. Robust Tests of Equality of Means FWAs, level of productivity of employees, and job performance of employees according to the market that the company serves.

\begin{tabular}{|c|c|c|c|c|c|}
\hline & & Statistica & df1 & df2 & Sig. \\
\hline Weekend work & Welch & 4.666 & 4 & 91.11 & 0.002 \\
\hline Work in shifts & Welch & 0.72 & 4 & 92.348 & 0.581 \\
\hline Overtime & Welch & 1.792 & 4 & 92.56 & 0.137 \\
\hline Part time job & Welch & 1.524 & 4 & 94.185 & 0.202 \\
\hline Flexible working hours & Welch & 6.657 & 4 & 92.065 & 0 \\
\hline Temporary employment & Welch & 5.158 & 4 & 92.502 & 0.001 \\
\hline Teleworking before COVID - 19 pandemic & Welch & 3.411 & 4 & 91.418 & 0.012 \\
\hline Teleworking during the COVID - 19 pandemic & Welch & 4.885 & 4 & 92.511 & 0.001 \\
\hline Teleworking after COVID - 19 pandemic & Welch & 6.983 & 4 & 93.272 & 0 \\
\hline The level of productivity of employees & Welch & 3.594 & 4 & 93.401 & 0.009 \\
\hline Job performance of employees & Welch & 2.13 & 4 & 91.341 & 0.083 \\
\hline
\end{tabular}

Source: Authors' calculation

Based on the conducted Robust Tests of Equality of Means, we conclude that in "Weekend work", "Flexible working hours", "Temporary work", "Teleworking before COVID-19 pandemic", "Teleworking during the COVID-19 pandemic"," Teleworking after the COVID-19 pandemic" and "The level of productivity of employees" there are statistically significant differences between respondents according to the market that the company serves because of the value of Sig. less than 0.05 . The post hoc test indicated that in "Weekend work" there are differences between the markets that companies serve, companies that serve the "global" market $(\mathrm{M}=2.79)$ use "weekend work" the least compared to companies that serve the "national" market $(\mathrm{M}=3.8)$ where "weekend work" is most applied. Firms serving "regional" markets use "Flexible working hours" the least $(\mathrm{M}=3.05)$ while companies serving "national market" use "flexible working hours" the most $(\mathrm{M}=4.33)$. Firms serving the "regional market" $(\mathrm{M}=2.93)$ use "Temporary work" the least, while firms serving the "national" market $(\mathrm{M}=3.95)$ use "temporary work" the most. Companies serving the "international" 
market used the least "Teleworking before the COVID-19 pandemic" $(\mathrm{M}=2.46)$ while companies serving the " $g$ lobal" market used the most "Teleworking before the COVID-19 pandemic), $(\mathrm{M}=3.25)$. Firms serving the "regional" market used the least "Teleworking during the COVID-19 pandemic" $(\mathrm{M}=3.38)$, while firms serving the "global market" used the most teleworking during the COVID-19 pandemic, $(\mathrm{M}=4.54)$. Companies serving the "regional" market $(\mathrm{M}=2.93)$ used the least "Teleworking after the COVID-19 pandemic" while companies serving the "global" market (M=4.25) used the most "teleworking after the COVID-19 pandemic".

Table 4. Spearman's correlation according to the level of application of FWAs, level of productivity of employees, and job performance of employees.

\begin{tabular}{|c|c|c|c|c|c|c|c|c|c|c|c|c|c|c|c|}
\hline & & & Mean & Stst. Deviation & Weekend work & \begin{tabular}{|l|l} 
Work in shitsts \\
\end{tabular} & Overtime & Part time job & 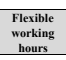 & $\begin{array}{l}\text { Temporary } \\
\text { employment }\end{array}$ & $\begin{array}{l}\text { Televorking befire } \\
\text { Covi } 19 \text { pandemic }\end{array}$ & $\begin{array}{l}\text { Telemorking during the } \\
\text { CovvD }-19 \text { pandemic }\end{array}$ & \begin{tabular}{|c} 
Teleworking after Covid \\
19 pandemic
\end{tabular} & 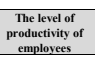 & 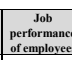 \\
\hline & Weekend work & & 3.22 & 1.464 & 1 & & & & & & & & & & \\
\hline & Work in shifts & & 3.27 & 1.602 & $.341^{* *}$ & 1 & & & & & & & & & \\
\hline & Overtime & Forralation Cofficicient & 3.74 & 1.219 & $.480^{* *}$ & $.424^{* *}$ & 1 & & & & & & & & \\
\hline & Part time job & Ererchation Coefficicint & 3.23 & 1.491 & $.522^{* *}$ & $.395^{* *}$ & $.409 * *$ & 1 & & & & & & & \\
\hline 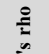 & Flexible working hours & Corralation Coefficicitut & 3.75 & 1.369 & $.432^{* * *}$ & $.218^{* *}$ & $.351^{* *}$ & $.553^{* *}$ & 1 & & & & & & \\
\hline$\frac{\bar{g}}{2}$ & Temporary employment & - Orralation Coefficicient & 3.45 & 1.395 & $.458^{* *}$ & $.406^{* *}$ & $.404 * *$ & $.538^{* * *}$ & $.430^{* *}$ & 1 & & & & & \\
\hline & $\begin{array}{c}\text { Teleworking before } \\
\text { covid - } 19 \text { pandemic }\end{array}$ & & 2.57 & 1.394 & $.208^{* *}$ & 0.028 & 0.025 & $.402^{* *}$ & .407*** & $.173^{*}$ & 1 & & & & \\
\hline & $\begin{array}{l}\text { Teleworking during the } \\
\text { CovID - } 19 \text { pandemic }\end{array}$ & -ormathion Confiricut & 4.01 & 1.267 & 0.044 & 0.064 & 0.089 & $.254^{* * *}$ & $.246^{* *}$ & $.193^{* *}$ & $.358^{* *}$ & 1 & & & \\
\hline & $\begin{array}{l}\text { Teleworking after } \\
\text { CoVID - } 19 \text { pandemic }\end{array}$ & ation Coefficicien & 3.76 & 1.337 & $.290^{* *}$ & 0.084 & $.228^{* *}$ & $.524 * *$ & $.560^{* *}$ & $.355^{* *}$ & $.568^{* *}$ & $.595^{* *}$ & 1 & & \\
\hline & $\begin{array}{l}\text { The level of productivity } \\
\text { of employes }\end{array}$ & 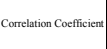 & 3.81 & 1.222 & $.275^{* *}$ & 0.015 & $.226^{* *}$ & $.264 * *$ & $.412^{* *}$ & 0.109 & $.222^{* *}$ & $.263^{* *}$ & $.382^{* *}$ & 1 & \\
\hline & $\begin{array}{l}\text { Job performance of } \\
\text { employees }\end{array}$ & Itation Coefficien & 4.595 & 0.4613 & $.150^{*}$ & 0.109 & 0.063 & . $196^{* * *}$ & $.286 * *$ & 0.075 & 0.029 & 0.078 & 0.104 & $.322^{* * *}$ & 1 \\
\hline
\end{tabular}

Source: Authors' calculation

Correlation represents the relationship between variables. The term correlation is most commonly applied in the context of a linear relationship between two continuous variables and is expressed as a Pearson correlation between product and moment. The Pearson correlation is typically used for common normally distributed data (data that accompany a bivariate normal distribution). While for abnormally distributed continuous data, regular data, or data with a relevant deviation, the Spearman rank correlation is used. Both of the above correlation coefficients are scaled to range from -1 to +1.0 indicates that there is no linear or monotonic association, and the relationship becomes stronger and eventually approaches a straight line (this refers to the Pearson correlation) and if the curve is constantly rising or falling (Spearman's correlation) as the coefficient approaches the absolute value of 1. Spearman's coefficient is usually abbreviated as $\rho$ (rho), or "rs" The strength of the correlation is measured as follows: If the correlation is: between $\mathbf{0 . 1 0}-\mathbf{0 . 2 9}$ it is weak (small), between 0.30 - 0.49 it is moderate and if the correlation is between $\mathbf{0 . 5 0}-\mathbf{1}$ it is strong (large). If the value of the coefficient is in the minus then that correlation is negative and if it has a positive sign then it is a positive correlation (Schober, Boer \& Schwarte, 2018). Based on the data analysis performed by Spearmn's correlation, it can be noticed that there is a weak positive relationship between "the level of productivity of employees" and "weekend work" (0.275), a weak positive relationship between "the level of productivity of employees" and "overtime" (0.226), weak positive relationship between "the level of productivity of employees" and "part-time work" (0.264), moderate positive relationship between" level of productivity of employees" and "flexible working hours" (0.412), weak positive relationship between "level of productivity of employees" and "teleworking before the COVID-19 pandemic" (0.222), a weak positive relationship between "level of productivity of employees" and "teleworking during the COVID-19 pandemic" (0.263), moderate positive relationship between "level of productivity of employees" and "teleworking after the COVID-19 pandemic" (0.382), weak positive relationship between "job performance of employees" and "weekend work" $(0.150)$, weak positive relationship between "job performance of employees" and "part-time work" (0.196), a weak positive relationship between "job performance of employees" and "flexible working hours" (0.286).

Based on the above results, the hypothesis is confirmed. 


\section{Discussion and Conclusion}

Flexible work arrangements are a necessary phenomenon of today's digital society. The accelerated pace of life, as well as the burden of work obligations, the emergence of unemployment, the economic crisis, the emergence of a pandemic, have led to workers agreeing to work flexibly to ensure the existence of their families. It is necessary to find a balance and use this type of work to increase employment and efficiency of the working-age population, to harmonize business and family obligations. Based on research by various authors such as Balau (1964), Golden (2001, 2009), Kelliher \& Anderson (2010), it was found that improved work performance can occur due to the application of flexible work arrangements and was a better relationship and communication between employees and employers. Employees have more flexibility in organizing their business obligations, remember to be freer to express their creativity, and in addition to their business obligations, they can also harmonize their privacy obligations. While the work of authors such as Konrad \& Mangel (2000) used Akerlof's (1982) theory that refers to the theory of gift exchange that has a positive effect on the provision of working life programs and worker productivity. This refers to a situation in which the employer provides a "gift" to the employee by paying him wages, as well as some other forms of benefits above those required by the market and the employees, return the "gift" in terms of performance above the norm. Based on the research, we found that the application of FWAs have a positive effect on worker productivity when using FWAs, employees work harder because they have more opportunities to do the job they work on. De Menezes \& Kelliher (2016) in their paper emphasized one important message for managers and it is that the role of FWAs can have a positive impact on organizational results. Workers will be more motivated to work, will invest more effort, the company's success will be greater and thus the satisfaction of both employees and employers will be at a high level because the goal of each company is to remain competitive in the market, beat competition, expand its business and to other segments and of course make a profit. The author of the paper Stavrou (2005), who examined the relationship between flexible work arrangements and organizational competitiveness, found that the results tend to support the use of flexible work arrangements and their perception of the positive impact on competitiveness in the European Union. Findings of a study by Bhattachary et al. (2005) suggest to managers that investing in flexible skills and employee behavior can increase the financial performance of a company. The work of the author Austin-Egole et al. (2020) indicates that the arrangements are focused on the benefits of employees who are motivated to invest more effort and that this will produce a positive effect on organizational performance. In the work of the author Berber \& Slavić (2019) which refers to the analysis of the impact of flexible work arrangements on the intentions of employees in Eastern Europe according to CRANET international research methodology, it is proven that the application of flexible work arrangements have a negative impact on employee intentions. Workers want to stay in the company they work for and want to advance in their business careers.

Empirical implications - The implementation of flexible work arrangements would be of great benefit in our country, as shown by research conducted based on completed questionnaires on the implementation of flexible work arrangements and their impact on corporate efficiency during the COVID - 19 pandemic. Employees should be acquainted with the advantages of applying this type of business to be able to organize their work in the best possible way, thus easing their work obligations and increasing efficiency to a higher level than at present.

The limitation of the research refers to a relatively small sample of respondents, the proposal for future research is an increased number of respondents who use flexible work arrangements in their business to keep the validity and results of the data at a higher level. If we want the organization to operate more efficiently, the essence is that each party, both employees and employers, be satisfied, and this option of applying flexible work arrangements can achieve that.

\section{REFERENCES}

Akerlof, G. A. (1982). Labor contracts as partial gift exchange. Quarterly Journal of Economics, 97(4), p. 543-569 DOI: https://doi.org/10.2307/1885099

Atkinson, C., \& Hall, L. (2009). The role of gender in varying forms of flexible working. Gender, Work and Organisation, 16(6), p. 650-666. DOI: https://doi.org/10.1111/j.1468-0432.2009.00456.x

Austin-Egole, I. S., Iheriohanma, E. B. J., \& Nwokorie, C. (2020). Flexible working arrangements and organizational performance: An overview. IOSR Journal of Humanities and Social Science (IOSR-JHSS), 25(5), p. 52 - 56. DOI: https://doi.org/10.9790/0837-2505065059

Azar, S., Khan, A., \& Van Eerde, W. (2018). Modeling linkages between flexible work arrangements' use and organizational outcomes. Journal of Business Research, 91, p. 134-143. DOI: https://doi.org/10.1016/j.jbusres.2018.06.004

Baltes, B. B., Briggs, T. E., Huff, J. W., Wright, J. A., \& Neuman, G. A. (1999). Flexible and compressed workweek schedules: A meta-analysis of their effects on work-related criteria. Journal of applied psychology, 84(4), p. 496. DOI: https://doi.org/10.1037/0021-9010.84.4.496

Barker, J. (1995). Family Ties: family-friendly policies are no longer a luxury, they are a competitive advantage. Sales and Marketing Management, 147, p. 18-24. 
Battisti, M., \& Vallanti, G. (2013). Flexible wage contracts, temporary jobs, and firm performance: Evidence from Italian firms. Industrial Relations: A Journal of Economy and Society, 52(3), p. 737-764. DOI: https://doi.org/10.1111/irel.12031

Beauregard, T. A., \& Henry, L. C. (2009). Making the link between work-life balance practices and organizational performance. Human resource management review, 19(1), p. 9-22. DOI: https://doi.org/10.1016/j.hrmr.2008.09.001

Berber, N., \& Slavić, A. (2019), Flexible Working Arrangements and Employee Turnover in the Central and Eastern Europe. Knowledge-Economy Society, p. 36. DOI: http://dx.doi.org/10.1080/09585192.2016.1277364

Berkery, E., Morley, M. J., Tiernan, S., Purtill, H., \& Parry, E. (2017). On the uptake of flexible working arrangements and the association with human resource and organizational performance outcomes. European Management Review, 14(2), p. 165-183. DOI: https://doi.org/10.1111/emre.12103

Bhattacharya, M., Gibson, D. E., \& Doty, D. H. (2005). The effects of flexibility in employee skills, employee behaviors, and human resource practices on firm performance. Journal of Management, 31(4), p. 622-640. DOI: https://doi.org/10.1177/0149206304272347

Blau, P. M. (1964). Exchange and power in social life. New York, NY: Wiley.

Boone, H. N., \& Boone, D. A. (2012). Analyzing Likert data. Journal of extension, 50(2), p. 1. Available on: https://joe.org/joe/2012april/tt2.php

Coenen, M., \& Kok, R. A. (2014). Workplace flexibility and new product development performance: The role of telework and flexible work schedules. European Management Journal, 32(4), p. 564-576. DOI: https://doi.org/10.1016/i.emj.2013.12.003

Conover, W. J., Johnson, M. E., \& Johnson, M. M. (1981). A comparative study of tests for homogeneity of variances, with applications to the outer continental shelf bidding data. Technometrics, 23(4), p. 351-361. DOI: https://doi.org/10.1080/00401706.1981.10487680

Dalton, D. R., \& Mesch, D. J. (1990). The impact of flexible scheduling on employee attendance and turnover. Administrative Science Quarterly, 35, p. 370-388.

De Menezes, L. M., \& Kelliher, C. (2016). Flexible Working, Individual Performance, and Employee Attitudes: Comparing Formal and Informal Arrangements. Human Resource Management, 56(6), p. 17. DOI: https://doi.org/10.1002/hrm.21822

Den Dulk, L., Groeneveld, S., Ollier-Malaterre, A., \& Valcour, M. (2013). National context in work-life research: A multilevel cross-national analysis of the adoption of workplace work-life arrangements in Europe. European Management Journal, 31, p. 478-494. DOI: https://doi.org/10.1016/j.emj.2018.01.007

Ferris, G. R., Arthur, M. M., Berkson, H. M., Kaplan, D. M., Harrell-Cook G., \& Frink, D. (1998). Toward a social context theory of the human resource management-organization effectiveness relationship. Human Resource Management Review, 8, p. 235-264

Golden, L. (2001). Flexible work schedules: Which workers get them? American Behavioural Scientist, 44(7), p. 11571178. DOI: https://doi.org/10.1177/00027640121956700

Golden, L. (2009). Flexible daily work schedules in US jobs: Formal introductions needed? Industrial Relations, p. 48(1), 27-54. DOI: https://doi.org/10.1111/j.1468-232X.2008.00544.x

Janssen, O., \& Van Yperen, N. W. (2004). Employees' goal orientations, the quality of leader-member exchange, and the outcomes of job performance and job satisfaction. Academy of Management Journal, 47(3), p. 375. DOI: https://doi.org/10.2307/20159587

Kelliher, C., \& Anderson, D. (2010). Doing more with less? Flexible working practices and the intensification of work. Human Relations, 63(1), p. 83-106 DOl: https://doi.org/10.1177/0018726709349199

Kim, T. K. (2015). T test as a parametric statistic. Korean Journal of Anesthesiology, 68(6), p. 540. DOI: https://doi.org/10.4097/kjae.2015.68.6.540

Klindžić, M., \& Marić, M. (2019). Flexible work arrangements and organizational performance-The difference between an employee and employer-driven practices. Društvena istraživanja, 28(1), p. 96. DOI: https://doi.org/10.5559/di.28.1.05 
Konrad, A. M., \& Mangel, R. (2000). The impact of work-life programs on firm productivity. Strategic Management Journal, 21, p. 1225-1237. DOI: https://doi.org/10.1002/1097-0266(200012)21:12<1225::AID-SMJ135>3.0.C0;2-3

Richardson, J., \& McKenna, S. (2014). Reordering spatial and social relations: A case study of professional and managerial teleworkers. British Journal of Management, 25, p. 724, DOI: https://doi.org/10.1111/1467-8551.12017

Schober, P., Boer, C., \& Schwarte, L. A. (2018). Correlation Coefficients. Anesthesia \& Analgesia, 126(5), p. 3. DOI: https://doi.org/10.1213/ane.0000000000002864

Stavrou, E. T. (2005). Flexible work bundles and organizational competitiveness: a cross-national study of the European work context. Journal of Organizational Behavior, 26(8), p. 923-947. DOI: https://doi.org/10.1002/job.356

Stavrou, E.T., Parry, E., \& Anderson, D. (2015). Nonstandard Work Arrangements and Configurations of Firm and Societal Systems. The International Journal of Human Resource Management, 26(19), p. 2412-2433, DOI: https://doi.org/10.1080/09585192.2014.992456

Stirpe \& Zárraga-Oberty, (2017). Are high-performance work systems always a valuable retention tool? The roles of workforce feminization and flexible work arrangements. European Management Journal, 35, p. 128-136. DOI: http://dx.doi.org/10.1016/j.emj.2016.04.002

Valverde, M., Tregaskis, O., \& Brewster, C. (2000). Labor flexibility and firm performance. International Advances in Economic Research, 6, p. 649-657.

Wright, P. M., \& Snell, S. A. (1998). Toward a unifying framework for exploring fit and flexibility in strategic human resource management. Academy of Management Review, 23, p. 756-773. 